PROCEEDINGS OF THE

AMERICAN MATHEMATICAL SOCIETY

Volume 139, Number 8, August 2011, Pages 2957-2972

S 0002-9939(2010)10713-X

Article electronically published on December 29, 2010

\title{
LINEAR REPRESENTATIONS OF SOLUBLE GROUPS OF FINITE MORLEY RANK
}

\author{
TUNA ALTINEL AND JOHN S. WILSON
}

(Communicated by Julia Knight)

\begin{abstract}
Sufficient conditions are given for groups of finite Morley rank having nontrivial torsion-free nilpotent normal subgroups to have linear representations with small kernels. In particular, centreless connected soluble groups of finite Morley rank with torsion-free Fitting subgroups have faithful linear representations. Along the way, using a notion of definable weight space, we prove that certain connected soluble groups of finite Morley rank with torsion-free derived subgroup can be embedded in groups of finite Morley rank whose Fitting subgroups have definable abelian supplements.
\end{abstract}

\section{INTRODUCTION}

Structural properties of groups of finite Morley rank are rich enough to provide a variety of challenging problems. The central interest in the analysis of these groups has been focused on the classification of the infinite simple groups. It was independently conjectured by Cherlin and Zil'ber that these are linear algebraic groups over algebraically closed fields.

This algebraicity conjecture suggests another important question, namely which groups of finite Morley rank have faithful linear representations (of finite dimension over fields). Not all groups of finite Morley rank have this property: direct products of algebraic groups in different characteristics and certain structures related to abelian varieties in algebraic geometry provide counterexamples, and there is the following elementary counterexample relevant to this paper: a divisible abelian group with $p$-torsion for infinitely many primes $p$ such that the $p$-ranks are all finite but unbounded. The nilpotent group of bounded exponent constructed by Baudisch [3] seems likely to be a counterexample, but no proof is known to the authors. All of the above examples are groups with large torsion subgroups.

On the other hand, there are results asserting that certain soluble groups of finite Morley rank are linear. Various field interpretation results in nonabelian soluble groups of finite Morley rank by Zil'ber [4, later work by Nesin and Enochs [7, 12. on centreless metabelian groups of finite Morley rank, and ongoing work of Frécon form a consistent line of development. Indeed, no counterexample to the following conjecture is known to the authors:

Received by the editors September 26, 2009 and, in revised form, July 20, 2010.

2010 Mathematics Subject Classification. Primary 03C60, 20F16.

The first author was partially supported by the ANR project "Groupes, Géométrie et Logique" JC05 47037:jaligot:eric.

Both authors acknowledge the support of FAW.

(c)2010 American Mathematical Society Reverts to public domain 28 years from publication 2957 
Conjecture. Let $G$ be a connected soluble group of finite Morley rank and let $K$ be the intersection of all normal definable subgroups $Y$ such that $G / Y$ is linear. Then $K$ is contained in the centre of $G$.

Examples mentioned above show that in general one need not have $K=1$. However the situation seems to be better for groups that do not have large torsion subgroups. In 2], we proved that all torsion-free nilpotent groups of finite Morley rank are linear groups. Here we study arbitrary groups of finite Morley rank with nontrivial torsion-free nilpotent normal subgroups. Our objective is to develop methods for finding linear representations with small kernels for such groups and thereby to prove that the subgroup $K$ in the Conjecture is small.

We shall prove that centreless connected soluble groups of finite Morley rank with torsion-free Fitting subgroups are linear groups. This follows from our main result:

Theorem 1. Let $G$ be a connected soluble group of finite Morley rank and suppose that $G$ has no infinite subgroups of finite exponent. Then $G$ has a linear representation with kernel $H$ contained in $Z_{3}(G)$.

We write $Z_{n}(G)$ for the $n$th term of the upper central series of a group $G$.

We cannot prove that the subgroup $H$ in Theorem 1 is definable, but it follows easily from Theorem 1 that the subgroup $K$ in the Conjecture satisfies $K \leq Z_{4}(G)$ for groups $G$ satisfying the hypotheses of the theorem; see Proposition 5.1 below.

If $G$ is a connected soluble group of finite Morley rank with Fitting subgroup $F$, then $G / F$ is abelian and there are nilpotent supplements to $F$ in $G$. On the other hand, as is shown by [9, Propositions 3.6 and 4.2], the existence of abelian supplements is equivalent to a difficult conjecture about fields of finite Morley rank. However, it may be true that $G$ always embeds in a group (interpretable in $G$ ) having abelian supplements to the Fitting subgroup. Our second theorem, needed for the proof of Theorem 1, provides some evidence for this:

Theorem 2. Let $G$ be a connected soluble group of finite Morley rank having a definable normal torsion-free nilpotent subgroup $N$ such that $G / N$ is abelian. Suppose that $G$ has no infinite subgroups of finite exponent. Then $G / C_{N}(G)$ embeds in a group $\widetilde{G}$ interpretable in $G$ with Fitting subgroup $\widetilde{N}$ and with the following properties:

(a) $N / C_{N}(G) \leq \widetilde{N}$;

(b) $\widetilde{G}$ has no infinite subgroups of finite exponent;

(c) $\widetilde{G}$ has a definable divisible abelian subgroup $\widetilde{T}$ such that $\widetilde{G}=\widetilde{N} \widetilde{T}$.

It follows in particular that if $G$ is a centreless connected soluble group of finite Morley rank having no infinite subgroups of finite exponent, then $G$ can be embedded in a group interpretable in $G$ that has an abelian supplement to its Fitting subgroup.

The proof of our theorems uses the Maltsev correspondence for torsion-free nilpotent groups, and also we have to introduce a definable version of the well-known notion of a weight space in representation theory. The prerequisite results are described fully in Section 2. In Section 3 we prove another linearity result that is not restricted to soluble groups, and Theorems 2 and 1 are proved in Sections 4 and 5 . 


\section{Preliminary Results}

In this section we recall some preliminary results and immediate consequences needed in our analysis. Our main general reference for groups of finite Morley rank is [4. All group and ring actions in this paper are on the right.

Groups of finite Morley rank. We recall that groups of finite Morley rank satisfy the descending chain condition on definable subgroups. Consequently each such group $G$ has a unique smallest definable subgroup of finite index, the connected component of $G$, denoted by $G^{\circ}$; if $G=G^{\circ}$, then $G$ is called connected. Another consequence of this condition is that every subset $X$ of a group of finite Morley rank is contained in a smallest definable subgroup, its definable hull. Since centralizers are definable, the descending chain condition also implies that each centralizer in a group of finite Morley rank is the centralizer of a finite subset. We will refer to this phenomenon as the chain condition on centralizers.

Lemma 2.1. Let $G$ be a group and let $L$ be a module for $G$ such that the pair $(G, L)$ is of finite Morley rank and $G$ is connected. Let $m_{1}, \ldots, m_{d} \in L$. Then there exists an integer $n$ with the following property: for each element $t$ of the augmentation ideal of the group ring $\mathbb{Z} G$, there exists an element $s \in \mathbb{Z} G$ which is a sum of at most $n$ elements of $\{g-1 \mid g \in G\}$ and satisfies $m_{i} t=m_{i}$ s for $1 \leq i \leq d$.

Proof. The componentwise action of $G$ on the $d$ th Cartesian power of $L$ is interpretable in $(G, L)$. Thus, it suffices to set $m=\left(m_{1}, \ldots, m_{d}\right)$ and apply Zil'ber's indecomposability criterion $([4, \S 5.4])$ to $\langle m(g-1) \mid g \in G\rangle$.

Suppose that $G$ is a torsion-free group of finite Morley rank. It is easy to prove that if $N$ is a definable normal subgroup, then $G / N$ is also torsion-free (see 4, §5.1, Exercise 13.b]) and, using similar methods, that $G$ is divisible. Thus $G$ has no proper subgroups of finite index and is connected. Moreover every element has a unique $n$th root for every $n \in \mathbb{N}^{*}$.

The following elementary lemma will be useful. It will allow us to ignore the divisible torsion subgroups in the Fitting subgroup of an ambient soluble group. This is crucial for the use of the Maltsev correspondence in Section 3.

Lemma 2.2. Let $G$ be a connected group of finite Morley rank and let $N$ be a definable nilpotent normal subgroup with divisible torsion subgroup. Then the group $N / C_{N}(G)$ is torsion-free.

Proof. Since the torsion subgroup $T$ of $N$ is divisible, it lies in the centre $Y$ of $N$ (for example from [17, Chapter 4]; see also [4, Corollary 6.12]), and moreover it has only finitely many elements of each finite order (from [4, §5.1, Exercise 7]). Therefore the centralizer of each element of $T$ has finite index in $G$ and so equals $G$ because $G$ is connected.

Fitting subgroup and Carter subgroups. Every group $G$ of finite Morley rank has a Fitting subgroup $F$, its unique maximal nilpotent normal subgroup. By results of Belegradek and Nesin, $F$ is a definable subgroup (see [4, Theorem 7.3]). By results of Zil'ber and Nesin, if $G$ is connected and soluble, then both $G / F$ and $G / F^{\circ}$ are divisible abelian groups (see [4, Theorem 9.21]).

An important ingredient in the analysis of groups of finite Morley rank is the notion of a Carter subgroup, a definable, connected, nilpotent subgroup of finite index in its normalizer. In this paper, we only need the existence and conjugacy 
of Carter subgroups in soluble groups of finite Morley rank, first proven in [16]. A consequence of their conjugacy is the following covering property: if $G$ is a connected soluble group of finite Morley rank and $N$ is a definable, normal subgroup such that $G / N$ is nilpotent, then $G=N C$ where $C$ is any Carter subgroup of $G$.

The following lemma is an easy consequence of some work of Frécon using Carter subgroups, more precisely of [8, Proposition 3.26]:

Lemma 2.3. Let $G$ be a connected soluble group of finite Morley rank with no infinite subgroups of finite exponent. Then the derived subgroup $G^{\prime}$ is torsion-free.

Commutative rings of finite Morley rank. These rings will play an important role. The following fact, proven in [2], is a consequence of results in [5], 6] and [18].

Fact 2.4 ([2, Lemma 2.2]). Let $\Lambda$ be a connected commutative local ring of finite Morley rank, with maximal ideal $J$. Then $\Lambda / J$ is an algebraically closed field. If $\Lambda / J$ has characteristic 0 , then there is a field $\mathfrak{K} \leq \Lambda$ such that $\Lambda=J+\mathfrak{K}$ and $J \cap \mathfrak{K}=\{0\}$. Moreover $\Lambda$ is finite-dimensional, regarded as a vector space over $\mathfrak{K}$.

The maximal ideal $J$ in the statement is the Jacobson radical. It is a definable ideal in the ring language, being the set of noninvertible elements. On the other hand, the field $\mathfrak{K}$ is not necessarily a definable subset of $R$; it is only interpretable, i.e. definable modulo a definable equivalence relation. This absence of definable splitting is at the heart of the difficulties to be overcome in Section 4 .

The Maltsev correspondence. The linear structures that we need in order to construct linear representations will be provided by the Maltsev correspondence, which associates nilpotent Lie algebras to certain nilpotent groups. Various accounts of this correspondence exist in the literature; we will use the version in [15, since it is well adapted for definability questions. Indeed, the model-theoretic essence of Fact 2.5 below is that the Lie algebra structure and the nilpotent group to which this algebra is associated are biinterpretable through the identity map.

The Maltsev correspondence associates with each torsion-free divisible nilpotent group $N$ a nilpotent Lie algebra $\mathcal{L}(N)$ over the field $\mathbb{Q}$. Since elements of a divisible torsion-free nilpotent group $N$ have unique $s$ th roots for all integers $s \neq 0$, for $g \in N$ and $\lambda \in \mathbb{Q}$ the power $g^{\lambda}$ can be uniquely defined by the equation $\left(g^{\lambda}\right)^{s}=g^{r}$, where $\lambda=r / s$ with $r, s \in \mathbb{Z}$ and $s \neq 0$. In general, a torsion-free nilpotent group of finite Morley rank is divisible with unique roots. As a result, torsion-free nilpotent groups of finite Morley rank form a natural setting for the Maltsev correspondence.

For the rest of Section 2 we fix an integer $c \geq 1$ and restrict attention to torsionfree divisible nilpotent groups of class at most $c$ and nilpotent Lie algebras over $\mathbb{Q}$ of class at most $c$. In Stewart 15 it is shown that there are words $\sigma_{c}(x, y), \pi_{c}(x, y)$ in the free group on $x, y$ such that if $N$ is one of our groups, then sum and Lie bracket operations can be defined in $N$ by $u+v=\sigma_{c}(u, v)$ and $[u, v]=\pi_{c}(u, v)$ for all $u, v \in N$, and multiplication by $\lambda \in \mathbb{Q}$ by $\lambda u=u^{\lambda}$, in such a way that the set $N$ acquires the structure of a Lie algebra over $\mathbb{Q}$. Thus from $N$, regarded as a pure group structure in the sense of model theory - an $\mathcal{L}_{G}$-structure where $\mathcal{L}_{G}$ is the language of groups - we obtain a Lie algebra $\mathcal{L}(N)$-an $\mathcal{L}_{\text {Lie }}$-structure where $\mathcal{L}_{\text {Lie }}=\left\{\delta_{\lambda}(\lambda \in \mathbb{Q}),[],+,\right\}$ - with the same underlying set. (Because we are working with groups of bounded class, we do not need to consider the "extended words' $\sigma, \pi$ constructed in [15].) Thus the identity map defines in $N$ a nilpotent $\mathbb{Q}$-Lie algebra structure $\mathcal{L}(N)$ with underlying set that of the group $N$. 
Similarly, there is a word $\mu_{c}(x, y)$ in the free Lie algebra on $x, y$ such that if $L$ is a Lie algebra $L$ (over $\mathbb{Q}$, nilpotent of class at most $c$ ), that is, a structure in the language $\mathcal{L}_{\text {Lie }}$ satisfying the necessary identities, then products and inverses can be defined in $L$ by $u v=\mu_{c}(u, v)$ and $u^{-1}=-u$ in such a way that the set $L$ becomes a torsion-free divisible nilpotent group $\mathcal{G}(L)$. Again the defining map is the identity. This yields the following main result in [15]:

Fact 2.5 ([15, Theorem 2.4.2]). Let $G$ and $H$ be divisible nilpotent torsion-free groups; let $L$ be a nilpotent Lie algebra over $\mathbb{Q}$. Then the following are true:

(a) $\mathcal{G}(\mathcal{L}(G))=G ; \mathcal{L}(\mathcal{G}(L))=L$.

(b) $H$ is a subgroup of $G$ if and only if $\mathcal{L}(H)$ is a subalgebra of $\mathcal{L}(G)$.

(c) $H$ is a normal subgroup of $G$ if and only if $\mathcal{L}(H)$ is an ideal of $\mathcal{L}(G)$.

(d) $\varphi: G \rightarrow H$ is a group homomorphism if and only if $\varphi: \mathcal{L}(G) \rightarrow \mathcal{L}(H)$ is a Lie homomorphism. The kernel of $\varphi$ is the same in both cases.

(e) If $H$ is a normal subgroup of $G$, then $\mathcal{L}(G / H)=\mathcal{L}(G) / \mathcal{L}(H)$.

Using (a), one can rewrite (b)-(e) starting from $L$ and applying $\mathcal{G}$.

We note in particular that, as pointed out in [2, Lemma $2.3(\mathrm{c})]$, since $N, \mathcal{L}(N)$ have exactly the same definable sets, they have the same Morley rank.

Lemma 2.6. Let $m \geq 2$, let $\mathfrak{K}$ be a field of characteristic 0 and let $L$ be a Lie subalgebra (of class at most $c$ ) of the algebra of $m \times m$ zero-triangular matrices over $\mathfrak{K}$. Then $\mathcal{G}(L)$ is isomorphic to the image of $L$ under the exponential map, given by $x \mapsto \sum_{j=0}^{m-1}(1 / j !) x^{j}$.

Proof. For each $u \in L$ write $u^{\sharp}=\exp u$. The word $\mu_{c}(x, y)$ is in fact the word arising in the Baker-Campbell-Hausdorff formula (but terminated after commmutators of weight c); see [15, p. 302]. From the basic property of this formula we have $\log \left(u^{\sharp} v^{\sharp}\right)=\mu_{c}(u, v)$ for all $u, v \in L$; see [15, Lemma 2.1.1]. Thus $u^{\sharp} v^{\sharp}=(u v)^{\sharp}$ for all $u, v$; in other words, the map $x \mapsto x^{\sharp}$ is a group isomorphism. (A similar argument shows that this is an isomorphism of $\mathfrak{K}$-groups, as defined below.)

$\mathfrak{K}$-groups. Suppose that $\mathfrak{K}$ is any field of characteristic 0 . If $L$ is a Lie algebra over $\mathfrak{K}$ and $N=\mathcal{G}(L)$, then for all $u$ in $N$ and $\lambda$ in $\mathfrak{K}$ we may define the power $g^{\lambda}$ in $N$ to be the multiple $\lambda g$ in $L$. The map $N \times \mathfrak{K} \rightarrow N$ defined in this way satisfies the axioms characterizing $\mathfrak{K}$-groups (or $\mathfrak{K}$-powered groups), described for example in Warfield [17, Chapters 10-12]. The formal definition of a $\mathfrak{K}$-group is given in Warfield [17, p. 86]: such a group is a nilpotent group with a power map $N \times \mathfrak{K} \rightarrow N$ satisfying the identities

$$
\begin{gathered}
g^{1}=g, \quad g^{\lambda_{1}+\lambda_{2}}=g^{\lambda_{1}} g^{\lambda_{2}}, \quad g^{\lambda_{1} \lambda_{2}}=\left(g^{\lambda_{1}}\right)^{\lambda_{2}}, \quad \text { for all } \lambda_{1}, \lambda_{2} \in \mathfrak{K}, g \in N, \\
\left(g^{\lambda}\right)^{h}=\left(g^{h}\right)^{\lambda} \quad \text { for all } \lambda \in \mathfrak{K}, g, h \in N,
\end{gathered}
$$

and the Hall-Petresco identities. An important example is the group $U_{m}(\mathfrak{K})$ of all $m \times m$ unitriangular matrices over $\mathfrak{K}$, for each integer $m \geq 2$; see [17, Corollary 10.25]. Quillen [13] proved that the Maltsev correspondence establishes a full bijection between nilpotent Lie algebras over $\mathfrak{K}$ and nilpotent $\mathfrak{K}$-groups; the results are stated in [17, Chapter 12]. For the reader's convenience we prove the only fact that we need: 
Lemma 2.7. Let $\mathfrak{K}$ be a field of characteristic 0 and let $L$ be a finite-dimensional Lie algebra over $\mathfrak{K}$. Then $\mathcal{G}(L)$ has the natural structure of a finitely generated $\mathfrak{K}$ group, with power operations defined from the Lie algebra structure of $L$ by $g^{\lambda}=\lambda g$. Moreover $\mathcal{G}(L)$ is isomorphic as a $\mathfrak{K}$-group to a subgroup of $U_{m}(\mathfrak{K})$ for some $m \geq 2$.

Proof. We need to check that the map $\mathcal{G}(L) \times \mathfrak{K} \rightarrow \mathcal{G}(L)$ satisfies the identities defining $\mathfrak{K}$-groups. By Ado's theorem (see [1] or [10, pp. 202-203]), for some $m$ there is an isomorphism $\theta$ from $L$ to a subalgebra $\bar{L}$ of the algebra of $m \times m$ zero-triangular matrices over $\mathfrak{K}$. Let $\bar{\theta}$ be the composite of $\theta$ and the exponential map. By Lemma 2.6 the group $\mathcal{G}(\bar{L})$ is isomorphic (as a group) to $\operatorname{im} \bar{\theta}$, which is a subgroup of $U_{m}(\mathfrak{K})$ and is evidently closed under the power operations since $(\exp \bar{l})^{\lambda}=\exp \lambda \bar{l}$. Because $\operatorname{im} \bar{\theta}$ satisfies all identities holding in the $\mathfrak{K}$-group $U_{m}(\mathfrak{K})$, it is a $\mathfrak{K}$-group. For $l \in L, \lambda \in \mathfrak{K}$ we have

$$
(\bar{\theta} l)^{\lambda}=(\exp \theta(l))^{\lambda}=\exp (\lambda \theta(l))=\exp \theta(\lambda l)=\bar{\theta}(\lambda l),
$$

so that $\bar{\theta}$ is an isomorphism of groups with power maps, and any identity holding in its image holds in its domain. Therefore $\mathcal{G}(L)$ is a $\mathfrak{K}$-group.

Let $X$ be a set spanning $\bar{L}$ as vector space and let $N_{1}$ be the smallest $\mathfrak{K}$-subgroup of im $\bar{\theta}$ containing $\exp X$. Then $X \subseteq \log N_{1}$, a Lie subalgebra of $\bar{L}$, and so we have $\log N_{1}=\bar{L}$ and $N_{1}=\exp \bar{L}=\operatorname{im} \bar{\theta}$. The result now follows.

Lemma 2.8. Let $\mathfrak{K}$ be a field of characteristic 0 and let $N$ be a nilpotent group such that $\mathcal{L}(N)$ has the structure of a finitely generated Lie algebra over $\mathfrak{K}$. Suppose that $G=N \rtimes T$ and that the action of $T$ on $\mathcal{L}(N)$ induced by conjugation in $G$ is $\mathfrak{K}$-linear. Then $G$ has a $\mathfrak{K}$-linear representation with kernel $C_{T}(N)$.

Proof. Fix an integer $m>0$. By Lemma 2.7 $N=\mathcal{G}(\mathcal{L}(N))$ is naturally a finitely generated $\mathfrak{K}$-group. Let $\Delta$ be the augmentation ideal of the group algebra $\mathfrak{K} N$, and let $I$ be the ideal of $\mathfrak{K} N$ generated by $\Delta^{m+1}$ and all elements

$$
u^{\lambda}-\left(1+\lambda(u-1)+\cdots+\left(\begin{array}{c}
\lambda \\
m
\end{array}\right)(u-1)^{m}\right) \quad \text { with } u \in N, \lambda \in \mathfrak{K} ;
$$

here

$$
\left(\begin{array}{l}
\lambda \\
j
\end{array}\right)=\frac{\lambda(\lambda-1) \ldots(\lambda-(j-1))}{j !}
$$

for $\lambda \in \mathfrak{K}$ and each integer $j>0$. By [17, Lemma 11.3], $\mathfrak{K} N / I$ is finite dimensional. Moreover if $m$ is large enough, then the obvious map from $N$ to $\mathfrak{K} N / I$ is an embedding. This follows from the proof of [17, Lemma 11.4]. We sketch a direct proof. Since $N$ is isomorphic to a $\mathfrak{K}$-subgroup of $U_{m}(\mathfrak{K})$ for some $m$ by Lemma 2.7, it will suffice to prove the result for the special case when $N=U_{m}(\mathfrak{K})$. Let $M$ be the ring $M$ of $m \times m$ matrices over $\mathfrak{K}$ and let $\varphi: \mathfrak{K} N \rightarrow M$ be the map induced by the identity on $N$. It is easy to check that $\varphi$ maps all elements $\left(u_{1}-1\right) \ldots\left(u_{m}-1\right)$ with $u_{1}, \ldots, u_{m} \in N$ and all elements $(*)$ to zero, and so $\varphi$ factors through $\mathfrak{K} N / I$. Since the composite of the map $N \rightarrow \mathfrak{K} N / I$ and the map $\mathfrak{K} N / I \rightarrow V$ has trivial kernel, so does the map $N \rightarrow \mathfrak{K} N / I$.

Now let $N$ act on $\mathfrak{K} N$ by right multiplication, and $T$ by conjugation; it is well known that these actions give an action of $G$ on $\mathfrak{K} N$, defined by $x \cdot(n t)=(x n)^{t}$ for $x, n \in N, t \in T$. Here is the verification that this is a group action: for $x, n_{1}, n_{2} \in N$ 
and $t_{1}, t_{2} \in T$ we have

$$
\begin{aligned}
x .\left(\left(n_{1} t_{1}\right)\left(n_{2} t_{2}\right)\right) & =x \cdot\left(n_{1} n_{2}^{t_{1}^{-1}} t_{1} t_{2}\right)=\left(x n_{1} n_{2}^{t_{1}^{-1}}\right)^{t_{1} t_{2}} \\
& =\left(\left(x n_{1}\right)^{t_{1}} n_{2}\right)^{t_{2}}=\left(x \cdot\left(n_{1} t_{1}\right)\right) \cdot\left(n_{2} t_{2}\right),
\end{aligned}
$$

as required. Since the ideal $I$ is invariant under both the action by $N$ and the action by $T$, the action of $G$ on $\mathfrak{K} N$ induces an action on $\mathfrak{K} N / I$. If $n t$ is in the kernel, with $n \in N, t \in T$, then for all $x \in N$ we have

$$
x+I=(x+I) \cdot n t=(x n)^{t}+I .
$$

First taking $x=1$ we see that $n=1$, and then we conclude that $x+I=x^{t}+I$ for all $x \in N$ and hence that $t \in C_{T}(N)$.

\section{Interpretable Rings: The Aston theorem}

In this section, we prove a ring interpretation theorem and a linearity corollary that seem of wider interest. The residue fields of the interpretable local rings obtained in this section will be the underlying fields for the linear representations constructed in the paper. Our methods have their origins in 18 and make use of the Maltsev correspondence in a way reminiscent of [2]. However we need to utilize effectively the actions of groups on nilpotent normal subgroups and Lie algebras.

Theorem 3 (Aston theorem). Let $G$ be a connected (not necessarily soluble) group of finite Morley rank and let $N$ be a definable normal torsion-free nilpotent subgroup. Suppose that $N \not Z Z(G)$ and write $D=C_{N}(G)$.

(a) There exists a commutative ring $R$ interpretable in $G$ such that $\mathcal{L}(N / D)$ has the structure of a finitely generated $R$-module with $R$-bilinear Lie bracket on which the action of $G$ induced by conjugation in $G$ is $R$-linear.

(b) There are definable normal subgroups $P_{1}, \ldots, P_{n}$ of $G$ contained in $N$ with $\bigcap P_{i}=D$ and commutative local rings $R_{i}$ interpretable in $G$ with $R=$ $\bigoplus_{i=1}^{n} R_{i}$ such that $\mathcal{L}\left(N / P_{i}\right)$ is a finitely generated $R_{i}$-module with $R_{i}$ bilinear Lie bracket on which the induced action of $G$ is $R_{i}$-linear.

Proof. Since both $N$ and $D$ are torsion-free groups of finite Morley rank, they are divisible, and so the Lie algebras $\mathcal{L}(N), \mathcal{L}(D)$ and $\mathcal{L}(N / D)$ may be defined.

Let $L=\mathcal{L}(N / D)$ and let $E$ be the ring of group endomorphisms of $L$. We aim to construct a subring $R$ of $E$ that is interpretable in $G$.

The action of $G$ on $N$ by conjugation induces an action on $\mathcal{L}(N)$ (by Fact 2.5(d)), and so we may regard $\mathcal{L}(N)$ as a $G$-module. For any ascending chain $M_{1}<$ $M_{2}<\cdots$ of definable subgroups of $\mathcal{L}(N)$ the quotients $M_{i+1} / M_{i}$ are infinite divisible abelian groups; therefore the chain has length at most the Morley rank $d$ of $\mathcal{L}(N)$, and there are elements $m_{1}, \ldots, m_{d}$ of $\mathcal{L}(N)$ such that the definable hull of $\left\langle m_{1}, \ldots, m_{d}\right\rangle$ equals $\mathcal{L}(N)$. We apply Lemma 2.1 for $\mathcal{L}(N)$ and the set $\left\{m_{1}, \ldots, m_{d}\right\}$; let $n$ be the number given by Lemma 2.1 and let $U$ be the set of elements of $\mathbb{Z} G$ that are sums of at most $n$ elements of type $g-1$.

By the chain condition on centralizers there exist $k \in \mathbb{N}$ and elements $s_{1}, \ldots, s_{k} \in$ $G$ such that $D=C_{N}(G)=C_{N}\left(s_{1}, \ldots, s_{k}\right)$. For each $k$-tuple $u=\left(u_{1}, \ldots, u_{k}\right) \in$ $U^{(k)}$ let $\Phi(u, x, y)$ be the first-order formula

$$
\bigwedge_{i=1}^{k} x u_{i}=y\left(s_{i}-1\right) \text {. }
$$


We consider the following set:

$$
\Lambda=\left\{u \in U^{(k)} \mid(\forall x \in N)(\exists y \in N) \Phi(u, x, y)\right\} .
$$

Thus $\Lambda$ is interpretable in $G$. If $x_{1}, x_{2}$ are congruent modulo $D$, then by Fact 2.5(e), they are also congruent modulo $\mathcal{L}(D)$. Thus, $\left(x_{1}-x_{2}\right)(g-1)=0$ for all $g \in G$, and hence $\Phi\left(u, x_{1}, y\right)$ holds if and only if $\Phi\left(u, x_{2}, y\right)$ holds. Moreover if $y_{1}, y_{2} \in N$ and $\Phi\left(u, x, y_{1}\right), \Phi\left(u, x, y_{2}\right)$ both hold, then we have $y_{1}\left(s_{i}-1\right)=y_{2}\left(s_{i}-1\right)$ for each $i$, so that $y_{1}-y_{2}$ is centralized by $s_{1}, \ldots, s_{k}$ and hence is in $\mathcal{L}(D)$; thus $y_{1}$ and $y_{2}$ are congruent modulo $D$. It follows that each $u$ in $\Lambda$ induces a well-defined map $\bar{u}: L \rightarrow L$ and evidently $\bar{u} \in E$. We set $\Lambda_{1}=\{\bar{u} \mid u \in \Lambda\}$.

Claim 1. $\Lambda_{1}$ is a subgroup of $E$.

Proof of Claim 1. The set $\Lambda_{1}$ is nonempty since $\Lambda$ contains the $k$-tuples $(0, \ldots, 0)$ and $\left(s_{1}-1, \ldots, s_{k}-1\right)$ which represent the zero and identity maps. Let $u$ and $v$ be in $\Lambda$; we want to show that $\bar{u}-\bar{v} \in \Lambda_{1}$. Fix $i \in\{1, \ldots, k\}$. By the choice of $n$, there is an element $w_{i} \in U$ such that $m_{j} w_{i}=m_{j} u_{i}-m_{j} v_{i}$ for $j=1, \ldots, d$. The set $\left\{x \in \mathcal{L}(N) \mid x w_{i}=x u_{i}-x v_{i}\right\}$ is a definable subgroup containing $\left\{m_{1}, \ldots, m_{d}\right\}$ and so equals $\mathcal{L}(N)$. Therefore $w=\left(w_{1}, \ldots, w_{k}\right) \in \Lambda$ and $\bar{w}=\bar{u}-\bar{v}$.

We define

$$
\begin{aligned}
& \Lambda_{2}=\left\{\bar{u} \in \Lambda_{1} \mid\left(\forall \bar{v} \in \Lambda_{1}\right)\left(\bar{u} \bar{v} \in \Lambda_{1}\right)\right\}, \quad \Lambda_{3}=\left\{\bar{u} \in \Lambda_{2} \mid\left(\forall \bar{v} \in \Lambda_{2}\right)(\bar{u} \bar{v}=\bar{v} \bar{u})\right\}, \\
& R=\left\{\bar{u} \in \Lambda_{3} \mid(\forall x, y \in L)(\forall g \in G)((\bar{u}(x)) g=\bar{u}(x g)) \wedge(\bar{u}([x, y])=[\bar{u}(x), y])\right\} .
\end{aligned}
$$

Claim 2. $R$ is a commutative subring of $E$ and it is interpretable in $G$ (so has finite Morley rank). The Lie bracket in $L$ is $R$-bilinear and the elements of $G$ induce $R$-linear maps from $L$ to $L$.

Proof of Claim 2. Clearly each of the sets $\Lambda_{2}, \Lambda_{3}, R$ is an additive subgroup of $\Lambda_{1}$ containing the identity map. The conditions imposed above ensure that $R$ is closed for products, that its elements commute with each other, that the Lie bracket is $R$-bilinear, and that its elements commute with the action of $G$. Clearly $R$ is interpretable in $G$ and so has finite Morley rank.

Since finitely generated $R$-submodules are definable connected subgroups, any chain of such submodules has length at most the Morley rank of $(G, L)$. Hence $L$ is a finitely generated $R$-module. This completes the proof of assertion (a).

Since $R$ is a commutative ring of finite Morley rank, it is the direct sum of local rings $R_{1}, \ldots, R_{n}$, from [5, Theorem 2.6]. The ideals $R_{i}$ are definable in $R$ as the sets $e_{i} R$, where $e_{1}, \ldots, e_{n}$ are the idempotents that yield the direct sum decomposition. For each $i$ let $M_{i}$ be the largest $R$-submodule of $L$ that is annihilated by $R_{i}$. Thus each $M_{i}$ is definable, and it is easy to see that the map from $L$ to $\bigoplus L / M_{i}$ is an $R$-module isomorphism; in particular $\bigcap M_{i}$ is zero since it is annihilated by each $R_{i}$ and hence by $R$. For each $i$ let $P_{i} / D$ be the normal subgroup of $N / D$ associated with $M_{i}$ by the Maltsev correspondence. Since $G$ maps $M_{i}$ to itself, each $P_{i}$ is a normal subgroup of $G$ by Fact 2.5(d). Since $\bigcap M_{i}$ is the zero submodule of $L$, we have $\bigcap P_{i}=D$, and assertion (b) now follows.

Corollary 3.1. Let $G$ be a connected group of finite Morley rank and let $N$ be a definable torsion-free nilpotent normal subgroup. Let $D=C_{N}(G)$ and $E=$ $C_{G}(N / D)$. Then $G / E$ has a faithful linear representation. 
Proof. We use the notation of the proof of the Aston theorem. For each $i$, the group $G$ acts on $L / M_{i}$ as a group of $R_{i}$-linear maps; by Fact 2.4, $R_{i}$ is a finite-dimensional space over a field $\mathfrak{K}_{i}$, and so the image of $G$ is a linear group over $\mathfrak{K}_{i}$. The kernel of the action of $G$ on $L_{i}$ is equal to the kernel of the action on $N / P_{i}$ by Fact 2.5)(d) and so equals $C_{G}\left(N / P_{i}\right)$. Since $\bigcap_{i \leq n} C_{G}\left(N / P_{i}\right)=C_{G}(N / D)=E$, it follows that $G / E$ is linear over any field containing isomorphic images of all of the fields $\mathfrak{K}_{i}$.

\section{Abelian supplements}

Our objective in this section is to prove Theorem 2, We shall deduce it from a theorem concerning groups with structure like that of the groups $G / P_{i}$ given by the Aston theorem.

Assume that $G$ is a connected soluble group of finite Morley rank having no infinite subgroups of finite exponent, that $N$ is a definable normal torsion-free nilpotent subgroup, and moreover that $G=N C$ with $C$ a definable connected nilpotent subgroup. We let $L=\mathcal{L}(N)$ and we assume that there is a commutative local ring $R$ with $(G, R)$ of finite Morley rank such that $L$ is a finitely generated $R$-module with $R$-bilinear Lie bracket on which the action of $G$ (induced from the conjugation action on $N$ ) is $R$-linear. We shall prove the following result.

Theorem 4. There is a group $\widetilde{G}$, interpretable in $(G, R)$, with Fitting subgroup $\widetilde{N}$, and with the following properties:

(a) $G \leq \widetilde{G}$ and $N \leq \widetilde{N}$;

(b) $\widetilde{G}$ has no infinite subgroups of finite exponent;

(c) $\widetilde{G}$ has a definable divisible abelian subgroup $\widetilde{T}$ such that $\widetilde{G}=\widetilde{N} \widetilde{T}$.

We approach the proof through a series of lemmas. The quest for structures interpretable in $(G, R)$ will lead us to introduce the notion of a definable weight space and after Lemma 4.7 to use a construction related to the semisimple splitting construction explained in Segal [14, Chapter 7].

We shall consider the structure of $L$ as an $R C$-module. By a composition factor, we will mean a section $M_{1} / M_{2}$, with $M_{1}, M_{2}$ definable $R C$-submodules of $L$, such that $M_{1}$ is minimal with respect to strictly containing $M_{2}$. We write $J$ for the Jacobson radical of $R$ and $\mathfrak{K}$ for the field $R / J$.

Lemma 4.1. Let $X$ be a composition factor of $L$, regarded as an $R C$-module. The following assertions hold:

(a) $C^{\prime}$ acts trivially on $X$.

(b) Each element of $R C$ acts on $X$ like multiplication by an element of $\mathfrak{K}$.

Proof. The upper central series of $N$ corresponds under the Maltsev correspondence to a series $0<L_{1}<\cdots<L_{c}=L$ of definable $\mathbb{Q} G$-submodules of $L$. Since $R$ commutes with the action of $G$, each $L_{i}$ is an $R G$-submodule. Since $N$ acts trivially on each factor $L_{i} / L_{i-1}$ by Fact 2.5(d), (e) and since $G / N$ is abelian, $C^{\prime}$ acts trivially on each $L_{i} / L_{i-1}$. By the Jordan-Hölder theorem $X$ is isomorphic to a composition factor of some $L_{i} / L_{i-1}$, and (a) follows.

(b) Because $X$ is a composition factor, the ring $\mathfrak{K}_{1}$ of endomorphisms of $X$ as an $R C$-module is a field by Schur's lemma. Thus, $R$ being local, the kernel of the map from $R$ to $\mathfrak{K}_{1}$ must be the prime ideal $J$, and so the image of $R$ in $\mathfrak{K}_{1}$ is a field $\overline{\mathfrak{K}}$ isomorphic to $\mathfrak{K}$. Since $(X, R)$ has finite Morley rank, $X$ must have finite dimension 
over $\overline{\mathfrak{K}}$. Therefore $\mathfrak{K}_{1}$ is a finite field extension of $\overline{\mathfrak{K}}$. Since $\mathfrak{K}$ is algebraically closed, we conclude that $\overline{\mathfrak{K}}=\mathfrak{K}_{1}$, and (b) follows.

Definition 4.2. Let $X$ be a composition factor of $L$, regarded as an $R C$-module. By Lemma 4.1 (b), we have a homomorphism $\lambda_{X}: C \rightarrow \mathfrak{K}^{*}$ defined by $x c=\lambda_{X}(c) x$ for all $x \in X, c \in C$. We call $\lambda_{X}$ the weight of the module $X$ and denote it by $\operatorname{wt}(X)$. We call $\lambda$ a weight of $L$ if $L$ has a composition factor with weight $\lambda$.

Our next task is to introduce the weight spaces $L_{(\lambda)}$ of $L$.

Lemma 4.3. If $F$ is a field and $D$ is a nilpotent subgroup of upper triangular matrices in $\mathrm{GL}_{2}(F)$ containing a nonscalar diagonal matrix, then $D$ consists of diagonal matrices.

Proof. Suppose that $D$ contains the elements

$$
g=\left(\begin{array}{cc}
\lambda & 0 \\
0 & \mu
\end{array}\right) \text { and } h_{0}=\left(\begin{array}{cc}
\alpha & \beta \\
0 & \gamma
\end{array}\right)
$$

where $\lambda \neq \mu$, and define $h_{i+1}=\left[h_{i}, g\right]$ for all $i \geq 0$. An easy calculation shows that

$$
h_{n}=\left(\begin{array}{cc}
1 & \alpha^{-1} \beta\left(\lambda^{-1} \mu-1\right)^{n} \\
0 & 1
\end{array}\right)
$$

for all $n \geq 1$, and so since $D$ is nilpotent, we have $\beta=0$.

Lemma 4.4. The RC-module $L$ has only finitely many weights. For each weight $\lambda$ there is a submodule $L_{(\lambda)}$ of $L$ such that all composition factors of $L_{(\lambda)}$ and no composition factors of $L / L_{(\lambda)}$ have weight $\lambda$. Moreover $L$ is the direct sum of these $R C$-submodules.

Proof. Chains of finitely generated $R C$-submodules have length bounded by the Morley rank of $(G, R)$; thus $L$ has finite composition length and only finitely many weights.

Next suppose that $Y$ is an $R C$-module section of $L$ of composition length 2, with simple submodule $X$, and that $X, Y / X$ have different weights $\mu, \lambda$. We claim that $Y$ has an $R C$-module $W$ with $Y=X \oplus W$.

From Fact 2.4 the ring $R$ contains a field $\mathfrak{K}_{0}$ (not necessarily definable) with $R=J+\mathfrak{K}_{0}, J \cap \mathfrak{K}_{0}=\{0\}$ and hence $\mathfrak{K}_{0} \cong R / J=\mathfrak{K}$; thus $Y$ is a 2-dimensional vector space over $\mathfrak{K}_{0}$. Choose $c \in C$ with $\mu(c) \neq \lambda(c)$. The elements $\bar{\lambda}, \bar{\mu}$ of $\mathfrak{K}_{0}$ that map to $\lambda(c), \mu(c)$ are the eigenvalues of the map $\bar{c}$ induced by $c$ in $Y$. With respect to a basis of eigenvectors, the maps induced by the elements of $C$ have upper triangular matrices, as the eigenspace for $\bar{\mu}$ must be $X$, and so by Lemma 4.3 the matrices are diagonal. Hence $Y$ is a direct sum of two simple $\mathfrak{K}_{0} C$-submodules. Since the elements of $R$ induce $\mathfrak{K}_{0} C$-module endomorphisms, the two nonisomorphic simple $\mathfrak{K}_{0} C$-submodules are mapped to themselves by $R$ and so are $R C$-submodules.

The rest of the proof is a standard argument. Among the composition series $0=M_{0}<\cdots<M_{n}=L$ of $L$ as an $R C$-module, choose one for which the sum of the indices $i$ with $\operatorname{wt}\left(M_{i} / M_{i-1}\right)=\lambda$ is minimal. If for some $i$ we have $\operatorname{wt}\left(M_{i+1} / M_{i}\right)=$ $\lambda$ and $\operatorname{wt}\left(M_{i} / M_{i-1}\right) \neq \lambda$, then from the previous paragraph $M_{i+1} / M_{i-1}$ has a submodule $W / M_{i-1}$ with weight $\lambda$, and consideration of the composition series obtained by replacing $M_{i}$ by $W$ gives a contradiction. Thus some term of the series $\left(M_{i}\right)$ has the property required of $L_{(\lambda)}$. The second assertion now follows easily.

Lemma 4.5. If $\mu, \nu$ are weights, then $\left[L_{(\mu)}, L_{(\nu)}\right] \leq L_{(\mu \nu)}$. 
Proof. Of course we interpret $L_{(\mu \nu)}$ as zero if $\mu \nu$ is not a weight of $L$. Let $\left(M_{i}\right)_{i=0}^{m}$, $\left(N_{j}\right)_{j=0}^{n}$ be composition series of $L_{(\mu)}, L_{(\nu)}$ and define $W_{k}=\sum_{i+j=k}\left[M_{i}, N_{j}\right]$ for $0 \leq k \leq m+n$. Since $J$ annihilates each composition factor and the Lie bracket is $R$-bilinear, $J$ annihilates each quotient $W_{k} / W_{k-1}$. Let $c \in C$, and choose $r, s \in R$ with $r+J=\mu(c), s+J=\nu(c)$. For $i, j>0$ and $m \in M_{i}, n \in N_{j}$ we have $m c \equiv r m$ $\bmod M_{i-1}, n c \equiv s n \bmod N_{j-1}$ and so

$$
[m, n] c \equiv[m c, n c] \equiv[r m, s n] \equiv r s[m, n] \quad \bmod W_{i+j-1} .
$$

Thus if $k \geq 1$ and $w \in W_{k}$, then $w c \equiv(r s) w \bmod W_{k-1}$. Hence $c$ acts as multiplication by $r s+J=\mu(c) \nu(c)$ on any composition factor $U / V$ with $W_{k-1} \leq V \leq$ $U \leq W_{k}$. The result follows.

Now let $c \in C$, and define $c_{\mathrm{s}}$ to be the family of $R$-linear maps $t: L \rightarrow L$ with the following properties:

- $t$ maps each $L_{(\lambda)}$ to itself;

- $\left.t\right|_{L_{(\lambda)}}$ is multiplication by some element $r_{\lambda} \in R$ with $r_{\lambda}+J=\lambda(c)$;

- if $\mu, \nu$ and $\mu \nu$ are weights, then $r_{\mu \nu}=r_{\mu} r_{\nu}$;

- $\left.t\right|_{L_{(1)}}$ is the identity map.

Let $S$ be the family $1_{\mathrm{s}}$ corresponding to the identity element of $C$. Thus $S$ is a group of automorphisms and in particular its elements act as multiplications by elements of $1+J$ on each $L_{(\lambda)}$ and as the identity on $L_{(1)}$. Clearly each $c_{\mathrm{s}}$ is a coset of $S$. Let $T=\bigcup\left\{c_{\mathrm{s}} \mid c \in C\right\}$. Then $T$ is an abelian group, and the map $c \mapsto c_{\mathrm{s}}$ is a surjective homomorphism from $C$ to $T / S$.

Lemma 4.6. (a) If $t \in T$, then $t$ is a Lie algebra homomorphism of $L$.

(b) If $t \in c_{\mathrm{s}}$, then $\mathrm{ct}^{-1}$ acts as the identity on each composition factor of $L$ as an $R C$-module.

(c) The group $T$ is isomorphic to a subgroup of $\left(R^{*}\right)^{n}$, where $n$ is the number of distinct weights of $L$ (and $R^{*}$ denotes the group of units of $R$ ). Moreover all subgroups of $T$ of finite exponent are finite.

Proof. (a) Since $L$ is the sum of the weight spaces, it will suffice to prove that if $\mu$, $\nu$ are weights and $m \in L_{(\mu)}, n \in L_{(\nu)}$, then $[m, n] t=[m t, n t]$. Suppose that $t$ acts as multiplication by $r_{\mu}$ on $L_{(\mu)}$ and by $r_{\nu}$ on $L_{(\nu)}$; then it acts as multiplication by $r_{\mu} r_{\nu}$ on $\left[L_{(\mu)}, L_{(\nu)}\right]$ by Lemma 4.5 and the definition of $t$, and so for $m \in L_{(\mu)}$, $n \in L_{(\nu)}$ we have $[m, n] t=r_{\mu} r_{\nu}[m, n]=\left[r_{\mu} m, r_{\nu} n\right]=[m t, n t]$, as required.

(b) It suffices to note that if $X$ is a composition factor of $L_{(\lambda)}$, then both $c, t$ act on $X$ by multiplication by $\lambda(c)$.

(c) To prove the first assertion, we list the weights as $\lambda_{1}, \ldots, \lambda_{n}$ and consider the map from $T$ defined by $t \mapsto\left(r_{1}, \ldots, r_{n}\right)$, where $r_{i}$ is the element of $R$ such that $t$ acts on $L_{\left(\lambda_{i}\right)}$ as multiplication by $r_{i}$. By the definition of $T, r_{i}$ belongs to a coset of $1+J$ and thus lies in $R \backslash J$. Since $R$ is local, $r_{i} \in R^{*}$.

We note that the multiplicative group $1+J$ is torsion-free. Indeed, if $j \in$ $J^{d} \backslash J^{d+1}$, then for all $n \in \mathbb{Z} \backslash\{0\}$ we have $(1+j)^{n} \equiv 1+n j$ modulo $J^{d+1}$, and so $(1+j)^{n} \neq 1$ since $J^{d} / J^{d+1}$ has the structure of a vector space over $R / J$ and hence is a torsion-free additive group. Thus the kernel of the map $R^{*} \rightarrow \mathfrak{K}^{*}$ is torsion-free, and the second assertion in (b) follows since finite subgroups of $\mathfrak{K}^{*}$ are cyclic. 
Lemma 4.7. The image of $C \cap N$ in $L$ (under the map $N \rightarrow L$ given by the Maltsev correspondence) lies in $L_{(1)}$ and so is acted on trivially by $T$.

Proof. Let $\left(C_{i}\right)_{i=0}^{k}$ be any central series of $C$. Then $C$ acts trivially on each factor $\left(C_{i} \cap N\right) /\left(C_{i-1} \cap N\right)$ and so acts trivially on each factor $D_{i} / D_{i-1}$, where $D_{i}$ is the $\mathbb{Q}$-subalgebra of $L$ corresponding to $C_{i} \cap N$ in the Maltsev correspondence. Let $E_{i}$ be the $R C$-submodule generated by $D_{i}$. Since $G$ induces $R$-linear maps and $C$ acts trivially on $\left(E_{i-1}+D_{i}\right) / E_{i-1}$, it follows that $C$ acts trivially on each $E_{i} / E_{i-1}$. Hence all $R C$-composition factors of $E_{k}$ have weight 1 , and $D_{k} \leq E_{k} \leq L_{(1)}$.

The action of $T$ as Lie algebra automorphisms on $L$ induces an action as group automorphisms of $N$ by Fact 2.5(d). For $t \in T$ we write $t^{*}$ for the map $G \rightarrow G$ defined by

$$
(a c)^{t^{*}}=a^{t} c \quad \text { for all } a \in N, c \in C .
$$

Let $T^{*}=\left\{t^{*} \mid t \in T\right\}$.

Lemma 4.8. $\quad$ (a) $t^{*}$ is well-defined.

(b) $t^{*}$ commutes with the action of $C$ by conjugation on $N$.

(c) $t^{*}: G \rightarrow G$ is a homomorphism.

(d) $T^{*}$ is a subgroup of the automorphism group of $G$ and the map $t \mapsto t^{*}$ defines a group isomorphism from $T$ to $T^{*}$.

Proof. (a) If $a_{1} c_{1}=a_{2} c_{2}$ with obvious notation, then $c_{1} c_{2}^{-1}=a_{1}^{-1} a_{2} \in N \cap C$, so that $t$ acts trivially on $N \cap C$ by Lemma 4.7 therefore

$$
\left(a_{1}^{-1}\right)^{t} a_{2}^{t}=\left(a_{1}^{-1} a_{2}\right)^{t}=a_{1}^{-1} a_{2}=c_{1} c_{2}^{-1} \text { and } a_{1}^{t} c_{1}=a_{2}^{t} c_{2} .
$$

(b) The elements of $C$ induce automorphisms of $L$ taking each $L_{(\lambda)}$ to itself and the elements of $T$ act as multiplications by elements of $R$ on each $L_{(\lambda)}$; hence $t^{*}$ commutes with the automorphisms of $L$ induced by $C$ and so commutes with the action of $C$ on $N$.

(c) Let $a_{1} c_{1}, a_{2} c_{2} \in G$ with obvious notation. By (b) we have $\left(a_{2}^{c_{1}^{-1}}\right)^{t}=\left(a_{2}^{t}\right)^{c_{1}^{-1}}$, and so

$$
\begin{aligned}
\left(\left(a_{1} c_{1}\right)\left(a_{2} c_{2}\right)\right)^{t^{*}} & =\left(a_{1} a_{2}^{c_{1}^{-1}} c_{1} c_{2}\right)^{t^{*}}=a_{1}^{t}\left(a_{2}^{c_{1}^{-1}}\right)^{t} c_{1} c_{2} \\
& =a_{1}^{t}\left(a_{2}^{t}\right)^{c_{1}^{-1}} c_{1} c_{2}=\left(a_{1}^{t} c_{1}\right)\left(a_{1}^{t} c_{2}\right)=\left(a_{1} c_{1}\right)^{t^{*}}\left(a_{2} c_{2}\right)^{t^{*}} .
\end{aligned}
$$

(d) The definition gives $\left(t_{1} t_{2}\right)^{*}=t_{1}^{*} t_{2}^{*}$ for all $t_{1}, t_{2} \in T$ and (d) follows.

We now set $\widetilde{G}=G \rtimes T^{*}$ and address the definability issues for this group.

Lemma 4.9. (a) The group $T$ as a group of automorphisms of $L$ is interpretable in $(G, R)$. The action of $T$ on $L$ is interpretable in $(G, R)$.

(b) The group $T$ as a group of automorphisms of $N$ is interpretable in $(G, R)$. The action of $T$ on $N$ is interpretable in $(G, R)$.

(c) The group $T^{*}$ and its action on $G$ are definable in $(G, R)$. The isomorphism between $T$ and $T^{*}$ defined in Lemma 4.8(d) is interpretable in $(G, R)$.

(d) The group $\widetilde{G}$ is interpretable in $(G, R)$.

Proof. (a) The $R C$-module $L$ is the direct sum of finitely many weight spaces $L_{\left(\lambda_{1}\right)}, \ldots, L_{\left(\lambda_{n}\right)}$. Let $\Omega$ be the set of triples $(i, j, k)$ of indices with $\lambda_{i} \lambda_{j}=\lambda_{k}$. We first verify that the $\operatorname{cosets} c_{\mathrm{s}}$ form a uniformly definable family. Indeed, each coset 
$c_{\mathrm{s}}$ is an interpretable subset of $L \times L$ defined by the formula $\operatorname{coset}(x, y, c)$ where $\operatorname{coset}(x, y, z)$ is

$$
\begin{aligned}
& \exists r_{\lambda_{1}}, \ldots, r_{\lambda_{n}} \in R \\
& \quad\left(\bigwedge_{i=1}^{n}\left(r_{\lambda_{i}}+J=\lambda_{i}(z) \leftrightarrow\left(x \in L_{\left(\lambda_{i}\right)} \wedge y=r_{\lambda_{i}} x\right)\right) \wedge \bigwedge_{(i, j, k) \in \Omega} r_{\lambda_{i}} r_{\lambda_{j}}=r_{\lambda_{i} \lambda_{j}}\right) .
\end{aligned}
$$

Now $T$ is defined as $\{(x, y) \in L \times L \mid \exists c \in C \operatorname{coset}(x, y, c)\}$.

The action of $T$ on $L=\bigoplus L_{\left(\lambda_{i}\right)}$ is also interpretable in $(G, R)$. In other words, the group action map from $T \times L$ to $L$ is interpretable in $(G, R)$.

(b) This point follows from (a) and Lemma 4.6(a) using Fact 2.5(d). Indeed, the nilpotent group $N$ has the same underlying set as the Lie algebra $L$, and the graphs of the automorphisms of $N$ and $L$ induced by elements of $T$ are identical. The second statement holds for the same reason.

(c) This follows from (b) and the definition of $T^{*}$ and its action on $G$.

(d) This follows from points (b) and (c).

Let $\widetilde{N}$ be the Fitting subgroup of $\widetilde{G}$. Since the normalizer of $N$ contains $G, T^{*}$, we have $N \leq \widetilde{N}$. Moreover since $T^{*} \cong T$, by Lemma 4.6(b) all subgroups of $\widetilde{G}$ of finite exponent are finite. To complete the proof of Theorem 4 , it remains only to prove assertion (c).

Define

$$
U=\left\langle c t^{*-1} \mid c \in C, t \in c_{\mathrm{s}}\right\rangle .
$$

Lemma 4.10. The subgroup $N U$ is nilpotent and normal in $\widetilde{G}$.

Proof. Let $m$ be the maximum of the composition lengths of the weight spaces $L_{(\lambda)}$.

Since $T^{*}$ centralizes $C$, the inclusion $U \leq C T^{*}$ holds and the group $U$ is nilpotent. Therefore since $N$ is nilpotent and $N \triangleleft \widetilde{G}$, to prove that $N U$ is nilpotent it will suffice to prove that for all $u_{1}, \ldots, u_{m} \in U$ we have $\left[a, u_{1}, \ldots, u_{m}\right]=1$ for all $a \in N$. This requirement translates via Fact 2.5 (d) to the requirement that $\left(u_{1}-1\right) \ldots\left(u_{m}-1\right)$ acts as the zero map on $L$, and this in turn follows from the definition of the elements $t^{*}$ and Lemma 4.6 (a). Finally, since $\left[U, T^{*}\right]=1$, it follows that $N U \triangleleft \widetilde{G}$.

Proof of Theorem 4. We must establish assertion (c). From Lemma 4.10 we have $N U \leq \widetilde{N}$ and hence $\widetilde{G}=\widetilde{N} T^{*}$. The group $\widetilde{G}$, being interpretable in $(G, R)$, is of finite Morley rank. By construction, it is connected. Indeed, by Lemma 4.6(b) and the structure of abelian groups of finite Morley rank (Macintyre [11; cf. 4, Theorem 6.7]), $T$ is a divisible abelian group; thus $T^{*}$ is a divisible abelian group that is also interpretable in $(G, R)$.

Now we restrict our attention to $\widetilde{G}$ as a pure group of finite Morley rank in which $G$ embeds, not necessarily as a subgroup definable in $\widetilde{G}$. Let $\widetilde{T}$ be the definable hull of $T$ with respect to the pure group structure $\widetilde{G}$; then $\widetilde{T}$ too is divisible and abelian and hence is connected. Finally, $\widetilde{G}=\widetilde{N} \widetilde{T}$ since $\widetilde{G}=\widetilde{N} T^{*}$.

Proof of Theorem 2. Let $G$ satisfy the hypotheses of Theorem2, Write $D=C_{N}(G)$ and let $R, P_{1}, \ldots, P_{n}, R_{1}, \ldots, R_{n}$ be as given by the Aston theorem. Thus each pair $\left(G / P_{i}, R_{i}\right)$ is interpretable in $G$ and satisfies the hypotheses of Theorem 4 . We may take for $C$ any Carter subgroup of $G$. Therefore each $G / P_{i}$ can be embedded in a group $\widetilde{G}_{i}$ with Fitting subgroup $\widetilde{N}_{i}$ and with a definable divisible abelian subgroup 
$\widetilde{T}_{i}$ with the properties given by Theorem 4 . Since $D=\bigcap P_{i}$, the group $G / D$ embeds in the product $\widetilde{G}=\prod_{i \leq n} \widetilde{G}_{i}$. Evidently $\widetilde{G}$ and the subgroups $\widetilde{N}=\prod_{i \leq n} \widetilde{N}_{i}$, $\widetilde{T}=\prod_{i \leq n} \widetilde{T}_{i}$ have the properties required in Theorem 2 ,

\section{Proof of Theorem 1}

Before proving Theorem 1, the main linearity result of this paper, we note the following proposition, which shows how to obtain definable kernels of maps to linear groups from arbitrary kernels.

Proposition 5.1. Let $G$ be a connected group of finite Morley rank and let $H$ be the kernel of a linear representation $\theta$ over a field $\mathfrak{K}$. Define $Y$ by $Y / H=Z(G / H)$. Then the following are true:

(a) $Y$ is also the kernel of a linear representation of $G$ over $\mathfrak{K}$, and

(b) $Y$ is definable.

Proof. (a) Since $Y$ is the kernel of the action of $G$ on $G / H$ induced by conjugation, it is the kernel of an action on $\operatorname{im} \theta$ and on the finite-dimensional vector space over $\mathfrak{K}$ spanned by $\operatorname{im} \theta$.

(b) Let $K$ be the largest connected definable soluble subgroup contained in $H$. Since the image of $Z(G / K)$ under the natural map $G / K \rightarrow G / H$ lies in $Z(G / H)$, we have $Z(G / K) \leq Y / K$. But $[G, Y]$ is a connected definable subgroup by Zil'ber indecomposability (see [4, Theorem 5.26]) and is contained in $H$; hence $Y / K \leq$ $Z(G / K)$. Thus $Y / K=Z(G / K)$ and $Y$ is definable.

Proposition 5.2. Let $G$ be a connected soluble group of finite Morley rank, let $N$ be a definable torsion-free nilpotent normal subgroup, and suppose that $G$ has a definable divisible abelian subgroup $A$ such that $G=N A$. Then $G$ has a linear representation with kernel $K$ satisfying $K \cap N \leq Z(G)$.

Proof. Write $D=C_{N}(G)$. By assertion (b) of the Aston theorem, we can find definable normal subgroups $P_{i}$ with intersection $D$ and commutative local rings $R_{i}$ interpretable in $G$ such that the Lie algebra $L_{i}=\mathcal{L}\left(N / P_{i}\right)$ is an $R_{i}$-module with $R_{i}$-bilinear Lie bracket and $G$ acts $R_{i}$-linearly on $L_{i}$ for each $i$. By Fact 2.4, $R_{i}$ is a finite-dimensional vector space over a subfield $\mathfrak{K}_{i}$, and so $L_{i}$ is a finite-dimensional Lie algebra over $\mathfrak{K}_{i}$.

Fix an index $i$. Now $G / P_{i}$ is the product of the definable torsion-free subgroup $N / P_{i}$ and the definable abelian subgroup $A P_{i} / P_{i}$, and the intersection $W_{i} / P_{i}=$ $N / P_{i} \cap A P_{i} / P_{i}$ of these subgroups is definable and hence divisible. By the injective property of divisible abelian groups, there exists a (not necessarily definable) subgroup $B_{i} / P_{i}$ such that $A P_{i} / P_{i}=W_{i} / P_{i} \times B_{i} / P_{i}$. Hence we have $G / P_{i}=N / P_{i} \rtimes B_{i} / P_{i}$. From Lemma 2.8 this group has a linear representation over $\mathfrak{K}_{i}$ whose kernel $K_{i} / P_{i}$ satisfies $K_{i} \cap N \leq P_{i}$. Thus $G$ has a linear representation with kernel $K=\bigcap K_{i}$ over any field containing copies of all fields $\mathfrak{K}_{i}$, and $K \cap N \leq \bigcap P_{i}=D$, as required.

Proof of Theorem 1. Suppose that $G$ satisfies the hypotheses of Theorem 11. Let $N=G^{\prime}$. By 4, Corollary 9.9], $G^{\prime}$ is definable, connected and nilpotent, and from Lemma 2.3. $N$ is torsion-free. Therefore by Theorem 4 we can embed $G / C_{N}(G)$ in a group of the form $\widetilde{G}=\widetilde{N} \widetilde{T}$, with the properties and notation of that theorem. 
Let $E=C_{\widetilde{N}}(\widetilde{G})$. By Lemma 2.2, $\widetilde{N} / E$ is torsion-free and so $\widetilde{G} / E$ satisfies the hypotheses of Proposition 5.2 thus $\widetilde{G}$ has a linear representation whose kernel $H_{0}$ satisfies $H_{0} \cap \widetilde{N} \leq M$ where $M / E=C_{\widetilde{N} / E}(\widetilde{G} / E)$. Hence $\left[H_{0}, \widetilde{G}\right] \leq E \leq Z(\widetilde{G})$ and $H_{0} \leq Z_{2}(\widetilde{G})$. Write $H / C_{N}(G)=H_{0} \cap G / C_{N}(G)$. Then $H$ is the kernel of a linear representation of $G$ and $H \leq Z_{3}(G)$.

\section{ACKNOWLEDGEMENTS}

Part of the work reported here was carried out in Oberwolfach in April 2009 within the 'Research in Pairs' programme. The authors thank the organizers and staff of the MFO for their support and for providing perfect conditions for mathematical research. The authors also express their gratitude to Aston University (Birmingham, UK), where a significant part of the work was done in most congenial circumstances.

Le premier auteur remercie Olivier Frécon pour lui avoir expliqué avec patience les détails subtils de ses travaux.

The exposition of the results reported here has profited greatly from the comments of the referee on a previous version.

\section{REFERENCES}

1. I. D. Ado. The representation of Lie algebras by matrices. Uspehi Mat. Nauk. (N.S.) 6 (22) (1947), 159-173 (A.M.S Transl. No. 2 (1949)). MR0027753 (10:350c)

2. T. Altinel and J. S. Wilson. On the linearity of torsion-free nilpotent groups of finite Morley rank. Proc. Amer. Math. Soc. 137 (2009), 1813-1821. MR2470842 (2009m:03050)

3. A. Baudisch. A new uncountably categorical group. Trans. Amer. Math. Soc. 348 (1996), 3889-3940. MR.1351488 (96m:03020)

4. A. V. Borovik and A. Nesin. Groups of Finite Morley Rank. Oxford University Press, 1994. MR:1321141 (96c:20004)

5. G. Cherlin and J. Reineke. Categoricity and stability of commutative rings. Ann. Math. Logic 10 (1976), 367-399. MR0480007 (58:208)

6. I. S. Cohen. On the structure and ideal theory of complete local rings. Trans. Amer. Math. Soc. 59 (1946), 54-106. MR.0016094 (7:509h)

7. K. Enochs and A. Nesin. On 2-step solvable groups of finite Morley rank. Proc. Amer. Math. Soc. 110 (1990), 479-489. MR984788 (90m:03068)

8. O. Frécon. Sous-groupes de Hall généralisés dans les groupes résolubles de rang de Morley fini. J. Alg. 233 (2000), 253-286. MR 1793597 (2001k:20069)

9. O. Frécon. Splitting in solvable groups of finite Morley rank. J. of Logic and Analysis, to appear.

10. N. Jacobson. Lie Algebras, Dover Publications, 1962. MR0143793 (26:1345)

11. A. Macintyre. On $\omega_{1}$-categorical theories of abelian groups. Fund. Math. $\mathbf{7 0}$ (1971), 253-270. MR 0289280 (44:6471)

12. A. Nesin. On solvable groups of finite Morley rank. Trans. Amer. Math. Soc. 321 (1990), 659-690. MR968420 (91a:03073)

13. D. Quillen. Rational homotopy theory. Ann. of Math. (2) 90 (1969), 205-295. MR0258031 $(41: 2678)$

14. D. Segal. Polycyclic Groups. Cambridge Tracts in Mathematics, 82. Cambridge University Press, 1983. MR 713786 (85h:20003)

15. I. Stewart. An algebraic treatment of Mal'cev's theorem concerning nilpotent Lie groups and their Lie algebras. Compositio Math. 22 (1970), 289-312. MR0288158 (44:5356)

16. F. O. Wagner. Nilpotent complements and Carter subgroups in stable R-groups. Arch. Math. Logic 30 (1994), 23-34. MR1264277 (95f:03050) 
17. R. B. Warfield Jr. Nilpotent Groups. Lecture Notes in Math., 513. Springer-Verlag, 1976. MR0409661 (53:13413)

18. B. I. Zil'ber. Rings with $\aleph_{1}$-categorical theories. Algebra and Logic 13 (1974), 95-104 (translation from Algebra i Logika 13, No. 2 (1974), 168-187). MR0366650 (51:2897)

Institut Camille Jordan, Université Lyon 1, 43 Boulevard du 11 nOvembre 1918, 69622 Villeurbanne CEDEX, France

E-mail address: altinel@math.univ-lyon1.fr

University College, Oxford OX1 4BH, United Kingdom

E-mail address: wilsonjs@maths.ox.ac.uk 